\title{
Loss of RASSF1A Expression in Colorectal Cancer and Its Association with K-ras Status
}

\author{
Dan Cao, ${ }^{1}$ Ye Chen, ${ }^{1}$ Yuan Tang, ${ }^{2}$ Xing-Chen Peng, ${ }^{1}$ Hang Dong, ${ }^{1}$ Long-Hao Li, ${ }^{1}$ \\ Ke Cheng, ${ }^{1}$ Jun Ge, ${ }^{1}$ and Ji-Yan Liu ${ }^{1}$ \\ ${ }^{1}$ Department of Medical Oncology, Cancer Center, The State Key Laboratory of Biotherapy, West China Hospital, \\ West China Medical School, Sichuan University, 37 Guo Xue Xiang, Chengdu, Sichuan 610041, China \\ ${ }^{2}$ Department of Pathology, West China Hospital, West China Medical School, Sichuan University, Chengdu 610041, China
}

Correspondence should be addressed to Ji-Yan Liu; liujiyan1972@163.com

Received 6 April 2013; Accepted 29 May 2013

Academic Editor: Renfu Shao

Copyright (C) 2013 Dan Cao et al. This is an open access article distributed under the Creative Commons Attribution License, which permits unrestricted use, distribution, and reproduction in any medium, provided the original work is properly cited.

Background. The RAS-association domain family 1 A (RASSF1A) is a classical member of RAS effectors regulating cell proliferation and apoptosis. Loss of RASSF1A expression may shift the balance towards a growth-promoting effect without the necessity of activating K-ras mutations. Its potential association with K-ras mutations in colorectal cancer (CRC) is unclear. Methods. RASSF1A expression was examined in normal mucosa, adenoma, and tumor tissues of colon and rectum, respectively. We examined the association of RASSF1A expression, mutations of K-ras, and EGFR status in 76 primary CRCs. The relationship between clinicopathological characteristics and RASSF1A expression was also analyzed. Results. RASSF1A expression level decreased progressively in normal mucosa, adenoma and, tumor tissues, and the loss of RASSF1A expression occurred more frequently in tumor tissues. Of 76 primary CRCs, loss of RASSF1A expression and/or K-ras mutations were detected in $77 \%$ cases. Loss of RASSF1A expression was more frequent in K-ras wild-type than in mutation cases (63\% versus $32 \%, P=0.011)$. Conclusions. Our study indicates that loss of RASSF1A may be involved in pathogenesis of CRC, its expression was found predominantly in $\mathrm{K}$-ras wild-type CRCs, suggesting that it may be another way of affecting RAS signaling, in addition to K-ras mutations.

\section{Introduction}

Colorectal cancer (CRC) is one of the most commonly diagnosed malignancies worldwide and is still carrying a high morbidity and mortality. At least $50 \%$ of CRCs are thought to have a dysregulation of the RAS-RAF-MEKERK (also known as the mitogen-activated protein kinase, MAPK) pathway $[1,2]$. Among those molecules, K-ras plays an essential role in the initiation of MAPK pathway and has been widely established as an important oncogene since the first report about its mutation [3]. Approximately, onethird of CRCs have been reported to have activating K-ras mutations, which implied insensitivity to EGFR inhibitors. K-ras mutations are found mostly $(90 \%)$ in codons 12 and 13 but may also affect codon 61 and others [4]. Another mechanism, including mutations of BRAF [5] and NF1 [2], has also been reported to be involved in the overactive RAS signaling pathway.

The RAS-association domain family $1 \mathrm{~A}$ (RASSF1A) is a tumor suppressor gene located on chromosome 3p21.3 and is a member of a new group of RAS effectors thought to regulate cell proliferation and apoptosis [6]. RASSF1A has been shown to affect multiple cellular activities, including promotion of apoptosis, cell cycle arrest, and maintenance of genomic stability [7]. It was also reported to suppress the activated K-ras-induced oxidative DNA damage [8]. Mutations in RASSF1A are rare, and methylation is the major mechanism for RASSF1A inactivation. A direct correlation between promoter methylation and loss of RASSF1A expression has been shown in more than half of human cancers [9-12]. It is expressed in all nonmalignant epithelial cells, but not in a large variety of human cancers, including CRC, 
lung cancers, breast cancers, and ovarian carcinoma [1319] while, the exogenous expression of RASSF1A decreases colony formation in vitro and tumor formation in vivo [20]. Observations suggest that RASSF1A functions as a tumor suppressor through RAS-mediated apoptosis $[6,7]$. All these suggested that it may have a pivotal role in tumor prevention.

As implied by its designation, RASSF1A is thought to interact with K-ras through a RAS association domain that alters its effects. Loss of RASSF1A expression by methylation may shift the balance towards a growth-promoting effect without the necessity of activating K-ras mutations. Recently, several groups have reported upon the existence of a relationship between RASSF1A and RAS signaling way $[20,21]$. Although increasing evidence points to a direct binding between RASSF1A and K-ras, its association to and its effect on K-ras are still not decided [22]. Furthermore, EGFR is expressed in $80 \%$ of CRC, and several recent and concordant clinical studies have shown that EGFR status is independent of K-ras mutations in colorectal tumors [23]. However, whether RASSF1A expression is related to K-ras mutation, EGFR status, and clinical feature of CRCs still needs to be clarified.

In this study, in order to explore the role of RASSF1A in colorectal pathogenesis, expression of RASSF1A in normal mucosa, adenoma, and tumor tissues of colon and rectum was studied. Then, its association with clinicopathological characteristics was analyzed in primary CRCs, and its relationship between K-ras mutations and EGFR expression was also investigated.

\section{Materials and Methods}

2.1. Sample Collection. Eighty one formalin-fixed, paraffinembedded tissue samples from patients who underwent surgical resection for CRC during the period between October 2009 and July 2011 in our hospital were obtained. Only 76 samples with records of sufficient tumor tissue and accurate pathological staging were finally included in the study. Twenty of the normal epithelium retrieved up to $5 \mathrm{~cm}$ away from the tumor's edge, and 20 of the adenoma from the same patients were also evaluated in the clinical and histological study.

2.2. Histology. Fresh CRC samples were received after resection, fixed in $10 \% \mathrm{pH}$-neutral formalin, and embedded in paraffin. All the patients had the diagnosis of adenocarcinomas and were staged according to the American Joint Commission for Cancer staging (AJCC/TNM, the sixth version) system. Clinicopathological characteristics in our study included age, gender, tumor size, degree of histological differentiation (well/moderate/poor, WHO), depth of infiltration, and staging. All histological slides were reviewed by two senior pathologists from our institution to confirm the diagnoses and to evaluate the patterns of RASSF1A and EGFR. In the case of differing opinions, the definitive assessment was obtained by consensus.
2.3. Immunohistochemistry. Immunohistochemistry was performed using the un-avidin-biotin complex technique named EnVision and MaxVision. Four $\mu \mathrm{m}$ thick sections were cut consecutively from formalin-fixed, paraffin-embedded tissue. Sections were mounted on silanized slides and allowed to dry overnight at $37^{\circ} \mathrm{C}$. After deparaffinization and rehydratation, slides were incubated with $3 \%$ hydrogen peroxide solution for $5 \mathrm{~min}$. After a washing procedure with the supplied buffer, tissue sections were repaired for $40 \mathrm{~min}$ with ethylenediamine tetraacetic acid. The slides were incubated for $60 \mathrm{~min}$ at $37^{\circ} \mathrm{C}$ and then overnight at $4^{\circ} \mathrm{C}$ with mouse monoclonal anti-RASSF1A (mouse monoclonal, Abcam) and anti-EGFR (mouse monoclonal, Santa Cruz) at a dilution of $1: 30$ and $1: 300$. After three rinses in buffer, the slides were incubated with the secondary antibodies of RASSF1A (Polymerase antibody MaxVision, antimouse/rabbit, DakoCytomation) and EGFR (unbiotinylated antibody, EnVisionTM System, HRP, anti-mouse/rabbit, DakoCytomation). Tissue staining was visualized with a DAB substrate chromogen solution (DakoCytomation). Slides were counterstained with hematoxylin, dehydrated, and mounted. Each run included, for each patient, phosphate buffered solution (PBS) used as the primary antibody for the negative controls and normal epithelium known to express RASSF1A and EGFR served as the positive control.

Five fields of vision by high power lens $(\times 400)$ were selected randomly, and 200 cells were counted per field. Then, the percentage of positive cells was calculated. Nuclear and cytoplasmic reactivity for RASSF1A proteins was considered as positive or negative as described previously [24]. For RASSF1A:,$- 0 \%$;,$+ 1-30 \%$; ++, $>30 \%$; $\%$ indicates the percentage of the nuclear and cytoplasmic immunostained cells with each individual protein.

The percentage of labeled cells of EGFR expression was graded as follows: grade 0 , no positive cells; grade 1, 1$25 \%$ labeled tumor cells; grade 2, 25-50\% labeled tumor cells; grade $3,>50 \%$ positive tumor cells. The intensity of peroxidase deposits, ranging from light beige to dark brown, was assessed visually as indicating the tumor cell membrane, cytoplasm, or both and was scored as 0 (negative), 1 (weak), 2 (moderate), or 3 (strong). A composite score, potentially ranging from 0 to 9 , was obtained by multiplying the grade by the intensity [25]. Patients were analyzed as a function of their EGFR expression: low, $<6$ and high, $\geq 6$.

2.4. DNA Extraction from Paraffin Tissue Blocks. After identification of at least $75 \%$ tumor area by a pathologist, tumor tissue was manually dissected from five consecutive $10 \mu \mathrm{m}$ sections of the paraffin-embedded tissue. The extracted tumor cells were dissolved in a total volume of $190 \mu \mathrm{L}$ digestion buffer (DNA tissue mini kit, Qiagen) and were treated with proteinase $\mathrm{K}$ overnight at $56^{\circ} \mathrm{C}$. DNA purification was achieved using a nucleic acid robot device (BIO 101, Qiagen).

2.5. Detection of K-ras Codons 12 and 13 Mutations by Automatic Sequencing. PCR amplification was done in a total volume of $20 \mu \mathrm{L}$ containing $20 \mathrm{ng}$ genomic DNA, $0.2 \mathrm{mmol} / \mathrm{L}$ deoxynucleotide triphosphate, 0.5 units of Taq polymerase (HotStar Taq, Qiagen). The primer sets for codons 12 and 13 of 
TABLE 1: RASSF1A expression in normal tissue, adenoma, and tumor.

\begin{tabular}{|c|c|c|c|c|}
\hline \multicolumn{5}{|c|}{ RASSF1A } \\
\hline & Positive & Negative & $\chi^{2}$ & $P$ \\
\hline \multicolumn{5}{|c|}{ No. of cases (\%) } \\
\hline Normal tissue & $19(95 \%)$ & $1(5 \%)$ & & \\
\hline Adenoma & $14(70 \%)$ & $6(30 \%)$ & 15.135 & 0.001 \\
\hline Tumor & $37(49 \%)$ & $39(51 \%)$ & & \\
\hline
\end{tabular}

Bold values represent $P$ values which are considered to be statistically significant at $<0.05$.

the K-ras gene were $5^{\prime}$-AGGCCTGCTGAAAATGACTGAA$3^{\prime}$ (sense) and $5^{\prime}$-AAAGAATGGTCCTGC ACCAG-3' (antisense) flanking codons 12 and 13 . For DNA sequencing, PCR was performed in a total volume of $10 \mu \mathrm{L}$ containing the purified PCR products (20-50 ng), $1.6 \mathrm{pmol}$ primer, $1 \mu \mathrm{L}$ of BigDye terminator Mix, $1 \mathrm{x}$ adding buffer, and 0.1 units of Taq Polymerase. Amplification was carried out using one standard and one biotinylated primer. DNA isolated from the CRC cell line was used as positive control. In the negative controls, no DNA was added. Cycle sequencing analysis of PCR fragments was done with the BigDye Terminator system (PE Biosystems) using amplification primers for bidirectional sequencing. The reaction products were analyzed on an $\mathrm{ABI}$ PRISM 3700 sequencer (PE Biosystems).

2.6. Statistical Analysis. Statistical analyses were all performed using SPSS 13.0 software. To test for difference of RASSF1A expression among normal epithelium, adenoma, and cancer of colorectum, the chi-square analysis was performed for categorical variables. Associations between RASSF1A expression, K-ras mutation, EGFR status, and the clinicopathological parameters and the relationships among these three factors were analyzed using the Fisher's exact test (or chi-square test) for categorical variables.

\section{Results}

3.1. RASSF1A Expression in Normal Mucosa, Adenoma, and Colorectal Cancer. In this study, RASSF1A expression was detected in normal mucosa, adenoma, and tumor tissues of CRC patients. No patients had history of chemotherapy or radiotherapy before surgery. The pattern of RASSF1A protein expression was mixed nuclear/cytoplasmic staining. The positive expression of RASSF1A was found to be $95 \%(19 / 20)$ in the normal mucosa, $70 \%(14 / 20)$ in the adenoma, and $48.68 \%(37 / 76)$ in the tumor tissues, respectively. RASSF1A expression decreased progressively in the three groups, and the difference was significant $(P<0.001$ Table 1$)$. The loss of RASSF1A protein expression (51.32\%) was found to be more frequent in tumor tissues compared to the other two groups (Figure 1).

3.2. RASSF1A Expression and Patient Characteristics. In 76 CRC patients, forty-four were male, and the median age was $56 \pm 11.5(30-82)$. Of the 76 tumor tissues obtained, 38 cases $(50 \%)$ were located in the colon and 38 cases
(50\%) in the rectum. Forty-four cases (58\%) were well and moderately differentiated adenocarcinoma. Invaded depth of the majority (77\%) was T3 and T4. Of the all patients at the time of diagnosis, 20 had distant metastases including radical resectable liver metastases and palliative resectable lung metastases. Negative expression of RASSF1A occurred in 27 of 44 men (61\%) and in 12 of 32 women $(37 \%)$, and the difference was statistically significant $(P=0.040)$. In addition, loss of RASSF1A expression occurred more frequently in carcinoma of colon (24 of $38,63 \%)$ than in carcinoma of rectum $(15$ of $38,39 \%)(P=0.001$, Table 2$)$. The difference had no significance between RASSF1A expression and other clinical parameters such as age, tumor size, degree of histological differentiation, depth of infiltration, and stage $(P>0.05)$.

3.3. K-ras Mutation, EGFR Status, and Patient Clinicopathological Features. Twenty-eight (36\%) of the 76 CRC samples examined showed a mutation at either codons 12 or 13 of the K-ras gene. Of that 28, 26 (92\%) were at codon 12 and 3 (8\%) at codon 13; GGT-GTT Gly12Val, GGT-GAT Gly12Asp, and GGC-GAC Glyl3Asp were detected in this study (Figure 2). The difference of K-ras mutation had no significance in different age, sex, tumor size, degree of histological differentiation, and stage $(P>0.05)$, but $\mathrm{K}$-ras mutation was significantly associated with depth of infiltration $(P=0.015)$. Mutation rate appeared to be higher in T3/T4 (26 of 59, 44\%) than in $\mathrm{T} 1 / \mathrm{T} 2$ (2 of $17,12 \%)$.

According to the labeling-intensity scores, EGFR expression was considered high $(\geq 6)$ and low $(<6)$. High expression of EGFR was found to be $0 \%(0 / 20)$ in the normal mucosa, $5 \%(1 / 20)$ in the adenoma, and $18 \%(14 / 76)$ in the tumor tissues, respectively (Figure 3). The percentage of high EGFR expression increased progressively in the three groups, and the difference was significant $(P<0.05)$. There was no significant association between high expression of EGFR and such clinicopathological factors as age, gender, site, tumor size, degree of histological differentiation, and depth of infiltration $(P>0.05)$. But EGFR overexpression was associated with tumor stage, with the percentage of patients with EGFR overexpression was higher in TNM stage IV than in stages $\mathrm{I} / \mathrm{II} / \mathrm{III} \mathrm{CRCs}$ (33\% versus $12 \%$ respectively, $P=$ 0.023) (data not shown).

3.4. Association between Loss of RASSF1A Expression, K-ras Mutation, and EGFR Status. Overall, K-ras mutations were observed in 28 of 76 (37\%) and loss of RASSF1A was observed in 39 of $76(51 \%)$ cases. Of the 76 patients examined, loss of RASSF1A expression was found to have higher incidence in cases with K-ras wild-type (30 of $48,63 \%$ ) than in Kras mutation $(9$ of $28,32 \%)(P=0.011) .58$ of $76(77 \%)$ patients were observed to have loss of RASSF1A expression and/or K-ras mutations. For the 76 adenocarcinomas studied, $18(23 \%)$ had neither K-ras mutation nor loss of RASSF1A expression, and 9 (12\%) had both K-ras mutation and loss of RASSF1A expression. Neither the association between RASSF1A expression and EGFR status nor K-ras mutation and EGFR expression had significant difference $(P=0.895$, Table 3). 


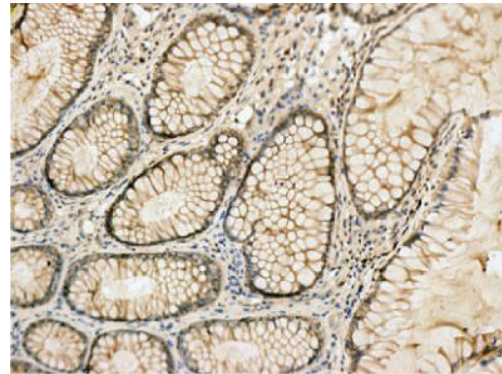

(a)

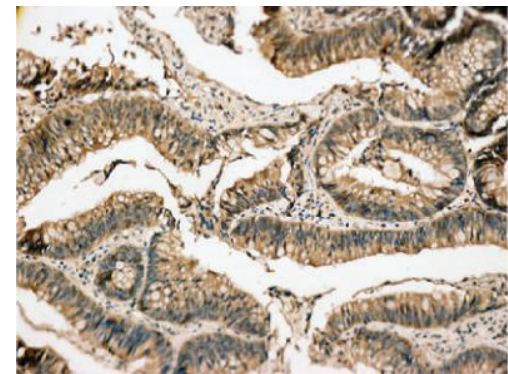

(b)

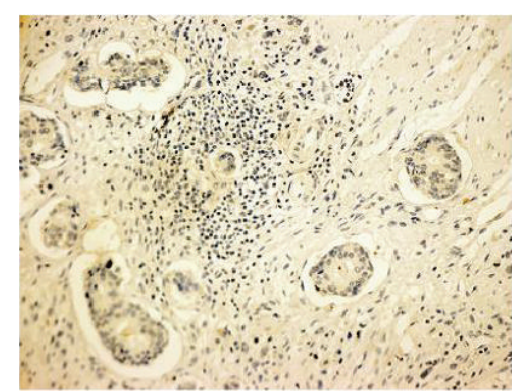

(c)

FIGURE 1: Immunohistochemical staining of RASSF1A in the normal tissue, adenoma, and tumor tissue. (a) Positive expression of RASSF1A in colonic epithelium (MaxVision, $\times 200$ ). (b) Typical mixed nuclear/cytoplasmic immunostaining of RASSF1A in adenoma (MaxVision, $\times 200$ ). (c) Negative expression of RASSF1A in colonic (or rectal) carcinoma (MaxVision, $\times 200$ ).

TABLE 2: Association between loss of RASSF1A expression and clinicopathological factors.

\begin{tabular}{|c|c|c|c|c|}
\hline \multirow{2}{*}{ Factors } & \multirow{2}{*}{$\begin{array}{c}\text { Total cases } \\
\text { No. }\end{array}$} & \multicolumn{3}{|c|}{ Loss of RASSF1A expression } \\
\hline & & No. (\% of total) & $\chi^{2}$ & $P$ \\
\hline \multicolumn{5}{|l|}{ Age } \\
\hline$<60$ & 41 & $22(53.66 \%)$ & 0.196 & 0.658 \\
\hline$\geq 60$ & 35 & $17(48.57 \%)$ & & \\
\hline \multicolumn{5}{|l|}{ Sex } \\
\hline Male & 44 & $27(61.36 \%)$ & 4.223 & 0.040 \\
\hline Female & 32 & $12(37.5 \%)$ & & \\
\hline \multicolumn{5}{|l|}{ Site } \\
\hline Colon & 38 & $24(63.16 \%)$ & 4.266 & 0.039 \\
\hline Rectum & 38 & $15(39.47 \%)$ & & \\
\hline \multicolumn{5}{|l|}{ Tumor size } \\
\hline$<5 \mathrm{~cm}$ & 55 & $31(56.36 \%)$ & 2.030 & 0.154 \\
\hline$\geq 5 \mathrm{~cm}$ & 21 & $8(38.10 \%)$ & & \\
\hline \multicolumn{5}{|c|}{ Differentiation grade } \\
\hline Poor & 32 & $13(40.63 \%)$ & 2.529 & 0.112 \\
\hline Moderate-well & 44 & $26(59.09 \%)$ & & \\
\hline \multicolumn{5}{|l|}{ Invasion depth } \\
\hline $\mathrm{T} 1+\mathrm{T} 2$ & 17 & $11(64.71 \%)$ & 1.572 & 0.210 \\
\hline $\mathrm{T} 3+\mathrm{T} 4$ & 59 & $28(47.58 \%)$ & & \\
\hline \multicolumn{5}{|l|}{ Tumor stage } \\
\hline I/II/III & 56 & $28(50 \%)$ & 0.114 & 0.736 \\
\hline IV & 20 & $11(55 \%)$ & & \\
\hline
\end{tabular}

Bold values represent $P$ values which are considered to be statistically significant at $<0.05$.

\section{Discussion}

In this study, we investigated expression of RASSF1A, K-ras mutation, and EGFR expression and analyzed the relationships between them in primary CRC in an attempt to understand the role of RASSF1A in RAS-mediated oncogenic transformation. The relationships between these factors and patients' clinicopathological characteristics were also analyzed.

In accordance with the major studies published to date $[26,27]$, our results showed that the incidence of positive RASSF1A expression decreased progressively in the normal mucosa, adenoma, and tumor tissues. The loss of RASSF1A protein expression was found to be more obvious in tumor tissues than in the nontumor tissues. High incidence of negative RASSF1A expression in carcinomas and an increased frequency in adenoma indicate that this may be an early event in colorectal carcinogenesis. As we know, the Ras signaling pathway is an essential mediator in the signaling that occurs in cells undergoing $\mathrm{CRC}$, which ultimately results in loss of cell-cell contacts, cytoskeletal remodeling, and increased mobility [28]. On the basis of many observations suggesting that RASSF1A mediates RAS-dependent apoptosis, it was hypothesized that RASSF1A inactivation is closely related to RAS activation in human cancers and thus contributes to malignant transformation by inhibiting RAS-mediated 


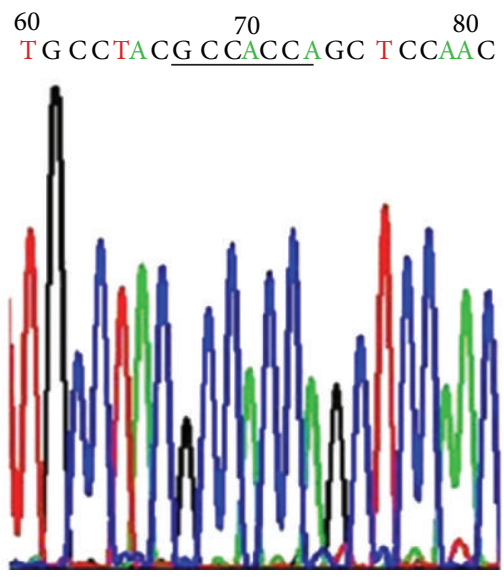

Wild-type

(a)

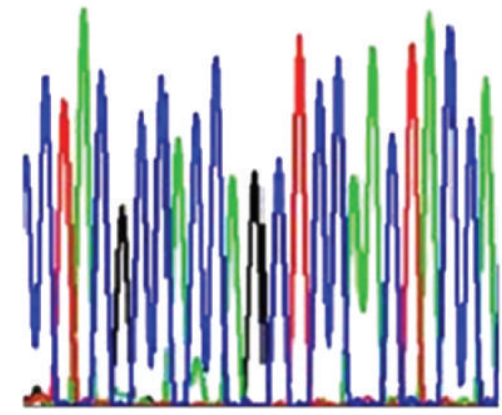

Gly12Val

(b)

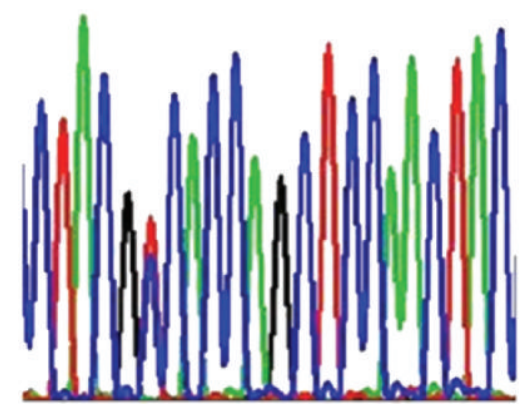

Gly13Asp

(c)

FIGURE 2: K-ras genotype in CRC. (a) K-ras wild-type. (b) Representative example of K-ras mutation of codon 12. (c) Representative results of K-ras mutation of codon 13.

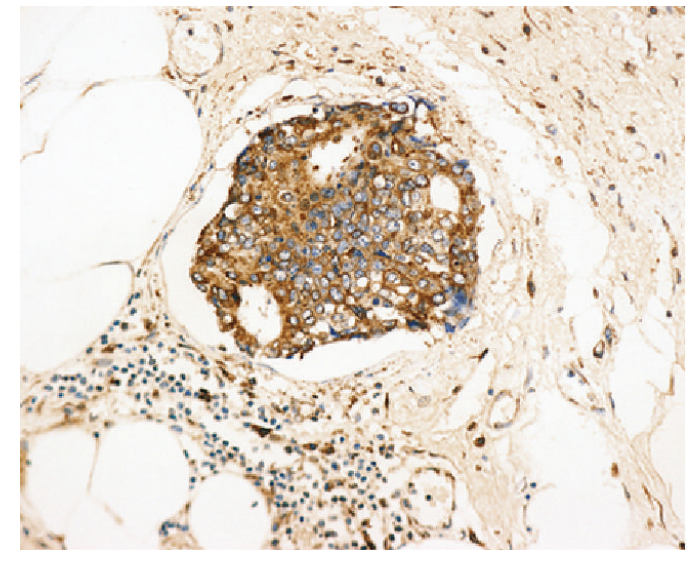

(a)

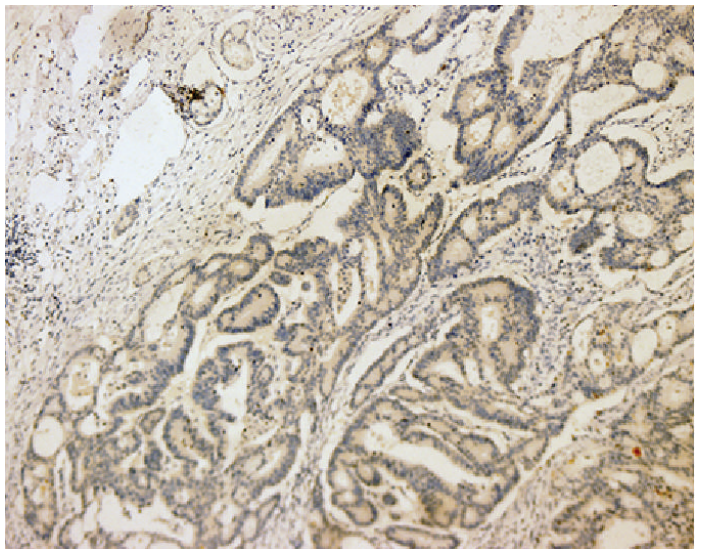

(b)

FIGURE 3: Expression of EGFR in CRC samples. (a) Typical immunoreactivity of membrane and cytoplasm of EGFR in CRC cells (EnVision, $\times 240$ ). (b) Negative expression of EGFR in CRC tissues (EnVision, $\times 100$ ).

TABLE 3: Relationship between loss of RASSF1A expression, K-ras mutation, and EGFR status in CRC.

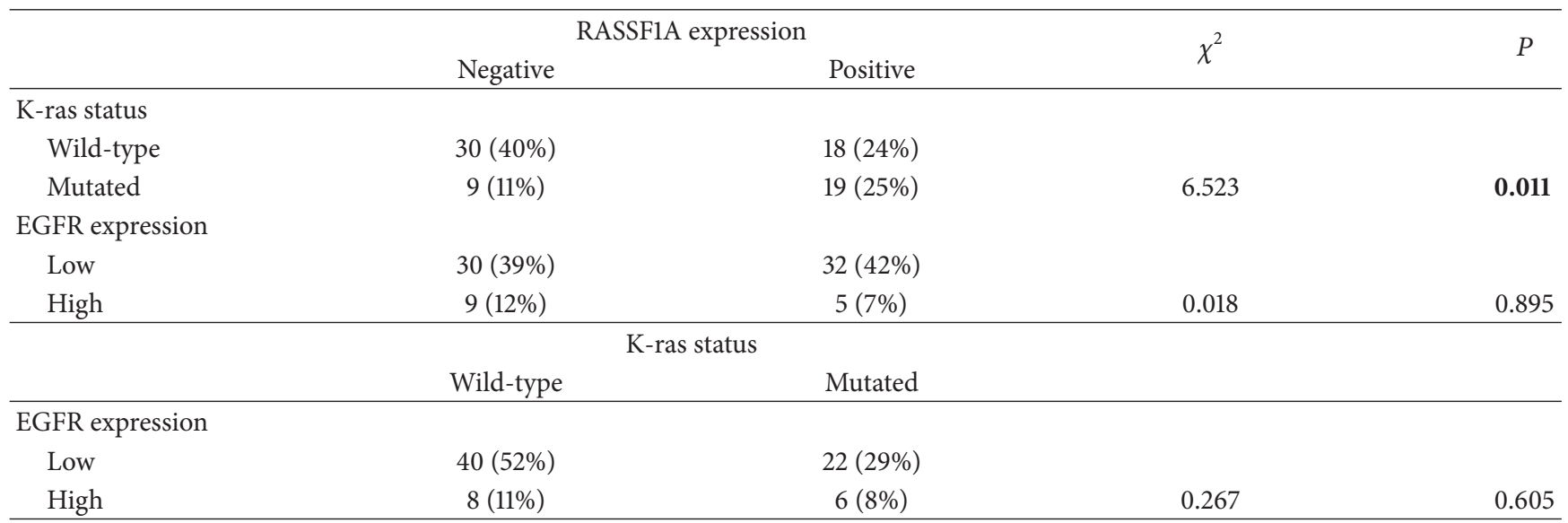

Bold values represent $P$ values which are considered to be statistically significant at $<0.05$. 
apoptosis [29]. Loss of RASSF1A expression could shift the balance towards a growth-promoting effect as a result of the loss of the proapoptotic and cell cycle-suppressive actions, without the necessity of RAS-activating mutations. Our results showed that the majority of the patients with colorectal cancers was observed to be with loss of RASSF1A expression and/or K-ras mutations. Among them, only a few of patients had both K-ras mutation and loss of RASSF1A expression. Unlike studies in lung cancer [20], in our data, significant potential association was found between loss of RASSF1A expression and K-ras mutation in 76 primary CRCs. The prevalence of the loss of RASSF1A expression in cases with the wild-type K-ras was higher than that in those with the mutant K-ras, and this difference was statistically significant, but the concrete mechanism was not clear. The CRC could be caused by combinatorial effects of various factors including gene mutations and environmental risk factors. K-ras mutations are one of the commonly believed mechanisms of CRC development. Otherwise, the loss of RASSF1A may act together with other risk factors to cause CRC without K-ras mutation. Without the loss of RASSF1A, these risk factors may not be sufficient to cause CRC, which may be the possible mechanism that the loss of RASSF1A is more frequent in K-ras wild-type CRCs. As we know, several data has shown that $\mathrm{K}$-ras mutation plays an important role in activating the RAS pathway in CRC. However, the exact mechanism of RASSF1A functioning as a RAS effector is not well elucidated. According to our data, we speculated that loss of RASSF1A expression might be a complementary mechanism in the onset of colorectal cancer in addition to K-ras mutations. Some researchers had found inactivation of RASSF1A and its synergistic effect with activated Kras in nasopharyngeal carcinoma [12]. In our study, $11.8 \%$ (9/76) of the samples were also found to be both loss of RASSF1A expression and K-ras mutations in CRC; it needs further studies to verify whether inactivation of RASSF1A has synergistic effect with activated K-ras in CRC.

In our study, we also found that frequency of loss of RASSF1A expression appeared to be higher in men compared with women and in carcinoma of colon than in rectum. The significance and the reason were not clear and still need further research and large size observations to clarify. In this series of 76 CRC patients, $36 \%$ of the malignant tumors were with K-ras mutated at either codons 12 or 13 . Of the mutated K-ras genes in these patients, $92 \%$ were mutated at codon $12,8 \%$ at codon 13 , and none of them was mutated at both codons 12 and 13. It was interesting to find that K-ras mutation rate appeared to be higher in T3/T4 than in T1/T2, and it probably indicated tumors with the tendency of invasion and metastasis. EGFR is overexpressed in many types of cancers, especially CRC, and the overexpression seems to reflect more aggressive histological and clinical behaviors. EGFR has been found to be elevated in CRCs, with expression rates ranging from 25 to $77 \%$ [30]. Our observations confirm that the rate of EGFR overexpression increased progressively from normal mucosa, adenoma to tumor tissues. In addition, we found that EGFR overexpression was associated with tumor stage, as the percentage of patients with EGFR overexpression was higher in TNM stage IV than in stages I/II/III CRCs. This possibly implied that patients with EGFR overexpression in advanced stage might have poor prognosis. In addition, we found that neither the association between RASSF1A expression and EGFR status nor K-ras mutation and EGFR expression had significant difference. Although another study had showed the inverse correlation of RASSF1A and EGFR in lung cancer [31], the similar phenomenon was not observed in CRC. It is possible that the discrepancy may stem from different tumor types or our limited samples. Further studies will be needed to address the questions.

In conclusion, the high frequency of loss of RASSF1A expression in carcinomas and an increased frequency in adenoma compared to normal tissue indicated that loss of RASSF1A expression might be an early event in CRC carcinogenesis. Importantly, the majority of the patients with colorectal cancers were observed to have K-ras mutations or/and loss of RASSF1A expression, and loss of RASSF1A expression was more frequently seen in K-ras wild-type cases. Thus, our results suggested that the loss of RASSF1A expression might be a complementary mechanism in the onset of colorectal cancer in addition to K-ras mutations.

\section{Authors' Contribution}

Dan Cao and Ye Chen contributed equally to this work.

\section{Acknowledgments}

The authors thank Dian-ying Liao for participating in immunohistochemistry staining. The authors also thank Yong-song Guan for reading the paper critically. This work was partly supported by the National Natural Science Foundation of China (81272457).

\section{References}

[1] J. Y. Fang and B. C. Richardson, "The MAPK signalling pathways and colorectal cancer," The Lancet Oncology, vol. 6, no. 5, pp. 322-327, 2005.

[2] T. Ahlquist, I. Bottillo, S. A. Danielsen et al., "RAS signaling in colorectal carcinomas through alterations of RAS, RAF, NF1, and/or RASSF1A," Neoplasia, vol. 10, no. 7, pp. 680-686, 2008.

[3] E. Santos, D. Martin Zanca, and E. P. Reddy, "Malignant activation of a K-ras oncogene in lung carcinoma but not in normal tissue of the same patient," Science, vol. 223, no. 4637, pp. 661664, 1984.

[4] S. Salerno, V. Bazan, R. M. Tomasino et al., "Specific codon 13 K-ras mutations are predictive of clinical outcome in colorectal cancer patients, whereas codon $12 \mathrm{~K}$-ras mutations are associated with mucinous histotype," Annals of Oncology, vol. 13, no. 9, pp. 1438-1446, 2002.

[5] S. Ogino, K. Nosho, G. J. Kirkner et al., "CpG island methylator phenotype, microsatellite instability, BRAF mutation and clinical outcome in colon cancer," Gut, vol. 58, no. 1, pp. 90-96, 2009.

[6] G. P. Pfeifer and R. Dammann, "Methylation of the tumor suppressor gene RASSF1A in human tumors," Biochemistry, vol. 70, no. 5, pp. 576-583, 2005. 
[7] L. B. Hesson, W. N. Cooper, and F. Latif, "The role of RASSF1A methylation in cancer," Disease Markers, vol. 23, no. 1-2, pp. 7387, 2007.

[8] S. H. Park, J. J. Kim, J. S. Chung et al., "RASSF1A suppresses the activated K-Ras-induced oxidative DNA damage," Biochemical and Biophysical Research Communications, vol. 408, no. 1, pp. 149-153, 2011.

[9] H. Donninger, M. D. Vos, and G. J. Clark, "The RASSF1A tumor suppressor," Journal of Cell Science, vol. 120, no. 18, pp. 31633172, 2007.

[10] D. G. Burbee, E. Forgacs, S. Zöchbauer-Müller et al., "Epigenetic inactivation of RASSF1A in lung and breast cancers and malignant phenotype suppression," Journal of the National Cancer Institute, vol. 93, no. 9, pp. 691-699, 2001.

[11] I. Kuzmin, J. W. Gillespie, A. Protopopov et al., "The RASSF1A tumor suppressor gene is inactivated in prostate tumors and suppresses growth of prostate carcinoma cells," Cancer Research, vol. 62 , no. 12, pp. 3498-3502, 2002.

[12] T. Wang, H. Liu, Y. Chen, W. Liu, J. Yu, and G. Wu, "Methylation associated inactivation of RASSF1A and its synergistic effect with activated K-Ras in nasopharyngeal carcinoma," Journal of Experimental and Clinical Cancer Research, vol. 28, no. 1, article 160, 2009.

[13] R. Bhagat, S. Chadaga, C. S. Premalata et al., "Aberrant promoter methylation of the RASSF1A and APC genes in epithelial ovarian carcinoma development," Cellular Oncology, vol. 35, no. 6, pp. 473-479, 2012.

[14] H. Chen, M. Suzuki, Y. Nakamura et al., "Aberrant methylation of RASGRF2 and RASSF1A in human non-small cell lung cancer," Oncology Reports, vol. 15, no. 5, pp. 1281-1285, 2006.

[15] S. K. Kee, J. Y. Lee, M. J. Kim et al., "Hypermethylation of the Ras association domain family 1A (RASSF1A) gene in gallbladder cancer," Molecules and Cells, vol. 24, no. 3, pp. 364-371, 2007.

[16] P. H. Y. Lo, D. Xie, K. C. Chan et al., "Reduced expression of RASSF1A in esophageal and nasopharyngeal carcinomas significantly correlates with tumor stage," Cancer Letters, vol. 257, no. 2, pp. 199-205, 2007.

[17] M.-G. Lee, H.-Y. Kim, D.-S. Byun et al., "Frequent epigenetic inactivation of RASSF1A in human bladder carcinoma," Cancer Research, vol. 61, no. 18, pp. 6688-6692, 2001.

[18] M. D. Vos, C. A. Ellis, A. Bell, M. J. Birrer, and G. J. Clark, "Ras uses the novel tumor suppressor RASSF1 as an effector to mediate apoptosis," Journal of Biological Chemistry, vol. 275, no. 46, pp. 35669-35672, 2000.

[19] W. Yeo, W.-L. Wong, N. Wong, B. K. Law, G. M. Tse, and S. Zhong, "High frequency of promoter hypermethylation of RASSF1A in tumorous and non-tumourous tissue of breast cancer," Pathology, vol. 37, no. 2, pp. 125-130, 2005.

[20] D.-H. Kim, J. S. Kim, J.-H. Park et al., "Relationship of Ras association domain family 1 methylation and K-Ras mutation in primary non-small cell lung cancer," Cancer Research, vol. 63, no. 19, pp. 6206-6211, 2003.

[21] B. Gao, X.-J. Xie, C. Huang et al., "RASSF1A polymorphism A133S is associated with early onset breast cancer in BRCA1/2 mutation carriers," Cancer Research, vol. 68, no. 1, pp. 22-25, 2008.

[22] J. M. A. Pijnenborg, G. C. Dam-de Veen, N. Kisters et al., "RASSF1A methylation and K-ras and B-raf mutations and recurrent endometrial cancer," Annals of Oncology, vol. 18, no. 3, pp. 491-497, 2007.
[23] E. Azizi, A. Kittai, and P. Kozuch, "Antiepidermal growth factor receptor monoclonal antibodies: applications in colorectal cancer," Chemotherapy Research and Practice, vol. 2012, Article ID 198197, 15 pages, 2012.

[24] X. Cui, Y. Shirai, T. Wakai, N. Yokoyama, S. Hirano, and K. Hatakeyama, "Aberrant expression of pRb and p16INK4a, alone or in combination, indicates poor outcome after resection in patients with colorectal carcinoma," Human Pathology, vol. 35, no. 10, pp. 1189-1195, 2004.

[25] N. S. Goldstein and M. Armin, "Epidermal growth factor receptor immunohistochemical reactivity in patients with American Joint Committee on Cancer Stage IV colon adenocarcinoma: implications for a standardized scoring system," Cancer, vol. 92, no. 5, pp. 1331-1346, 2001.

[26] X. Cui, T. Wakai, Y. Shirai, K. Hatakeyama, and S. Hirano, "Chronic oral exposure to inorganic arsenate interferes with methylation status of p16INK4a and RASSF1A and induces lung cancer in A/J mice," Toxicological Sciences, vol. 91, no. 2, pp. 372381, 2006.

[27] E. Miranda, A. Destro, A. Malesci et al., "Genetic and epigenetic changes in primary metastatic and nonmetastatic colorectal cancer," British Journal of Cancer, vol. 95, no. 8, pp. 1101-1107, 2006.

[28] G. Poulogiannis, F. Luo, and M. J. Arends, "RAS signalling in the colorectum in health and disease," Cell Communication and Adhesion, vol. 19, no. 1, pp. 1-9, 2012.

[29] M. Spugnardi, S. Tommasi, R. Dammann, G. P. Pfeifer, and D. S. B. Hoon, "Epigenetic inactivation of RAS association domain family protein 1 (RASSF1A) in malignant cutaneous melanoma," Cancer Research, vol. 63, no. 7, pp. 1639-1643, 2003.

[30] S. J. Cohen, R. B. Cohen, and N. J. Meropol, “Targeting signal transduction pathways in colorectal cancer: more than skin deep," Journal of Clinical Oncology, vol. 23, no. 23, pp. 53745385, 2005.

[31] N. Yanagawa, G. Tamura, H. Oizumi, M. Endoh, M. Sadahiro, and T. Motoyama, "Inverse correlation between EGFR mutation and FHIT, RASSFlA and RUNX3 methylation in lung adenocarcinoma: relation with smoking status," Anticancer Research, vol. 31, no. 4, pp. 1211-1214, 2011. 


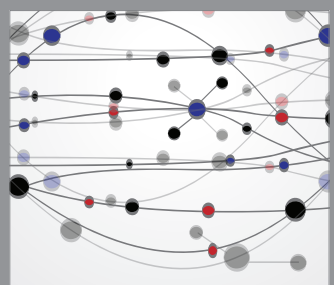

The Scientific World Journal
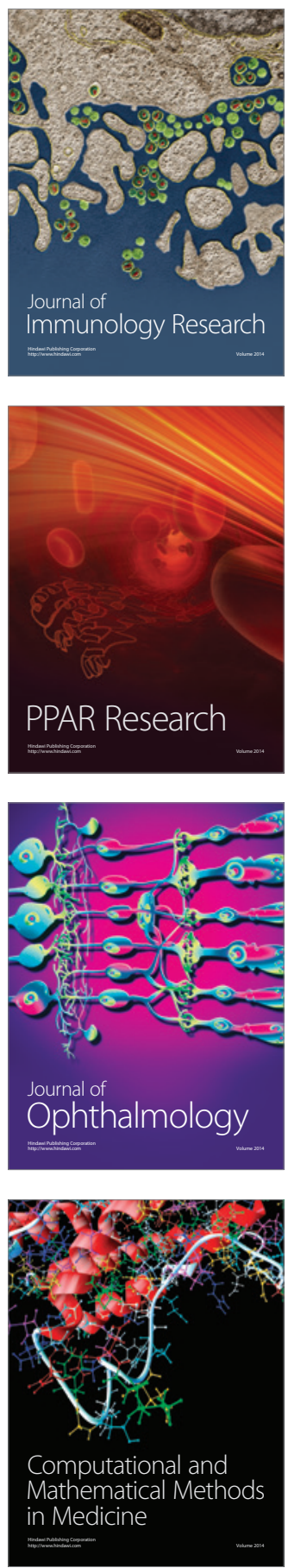

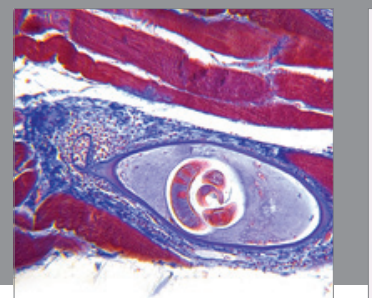

Gastroenterology

Research and Practice
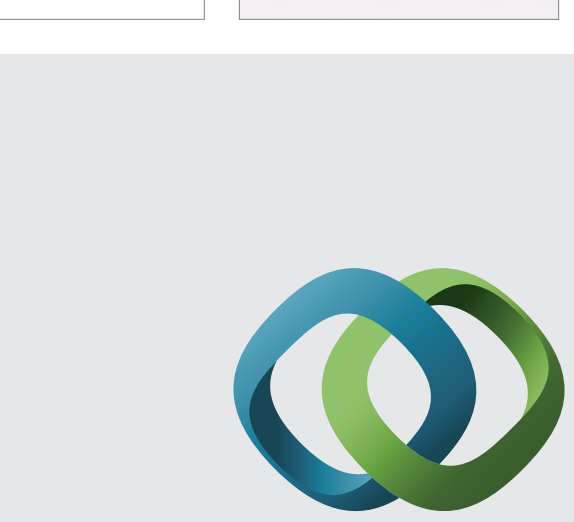

\section{Hindawi}

Submit your manuscripts at

http://www.hindawi.com
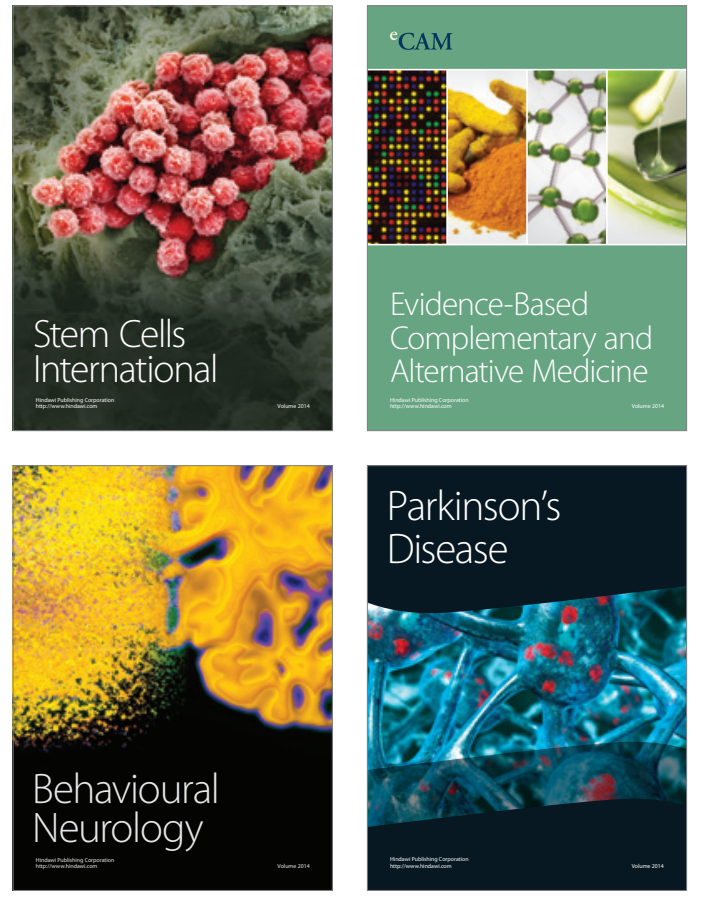
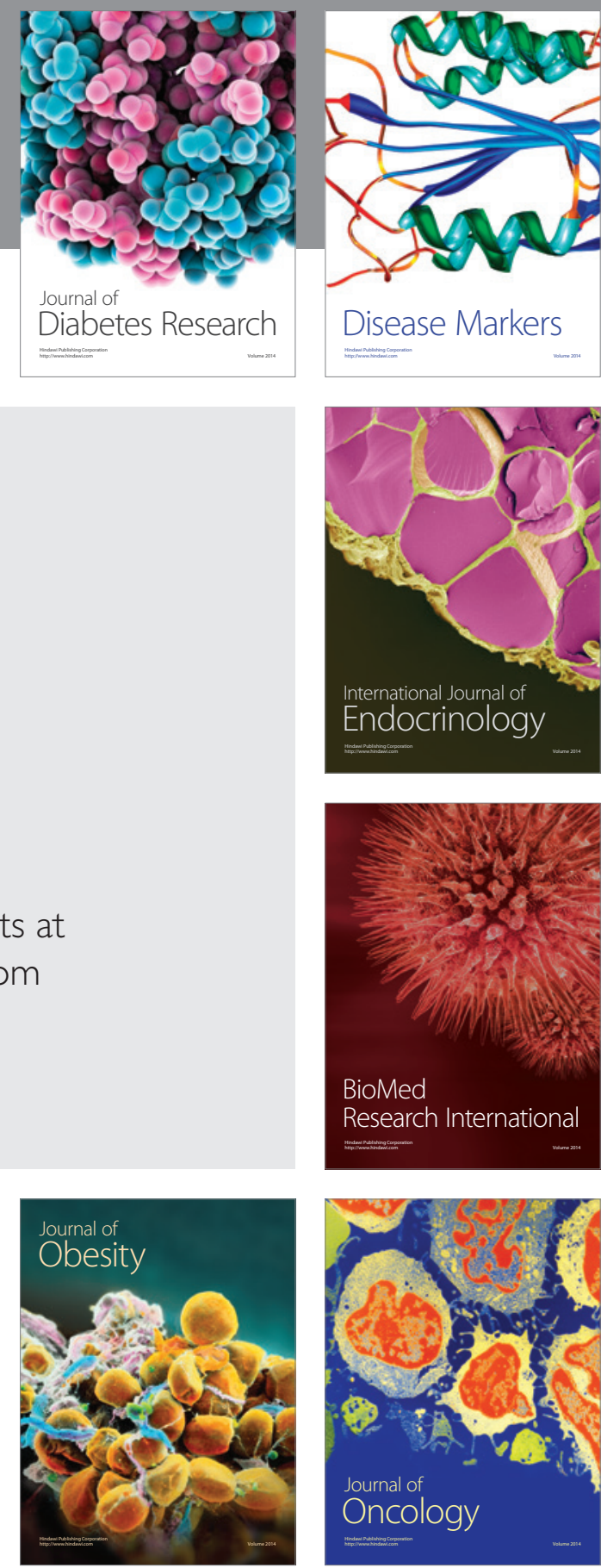

Disease Markers
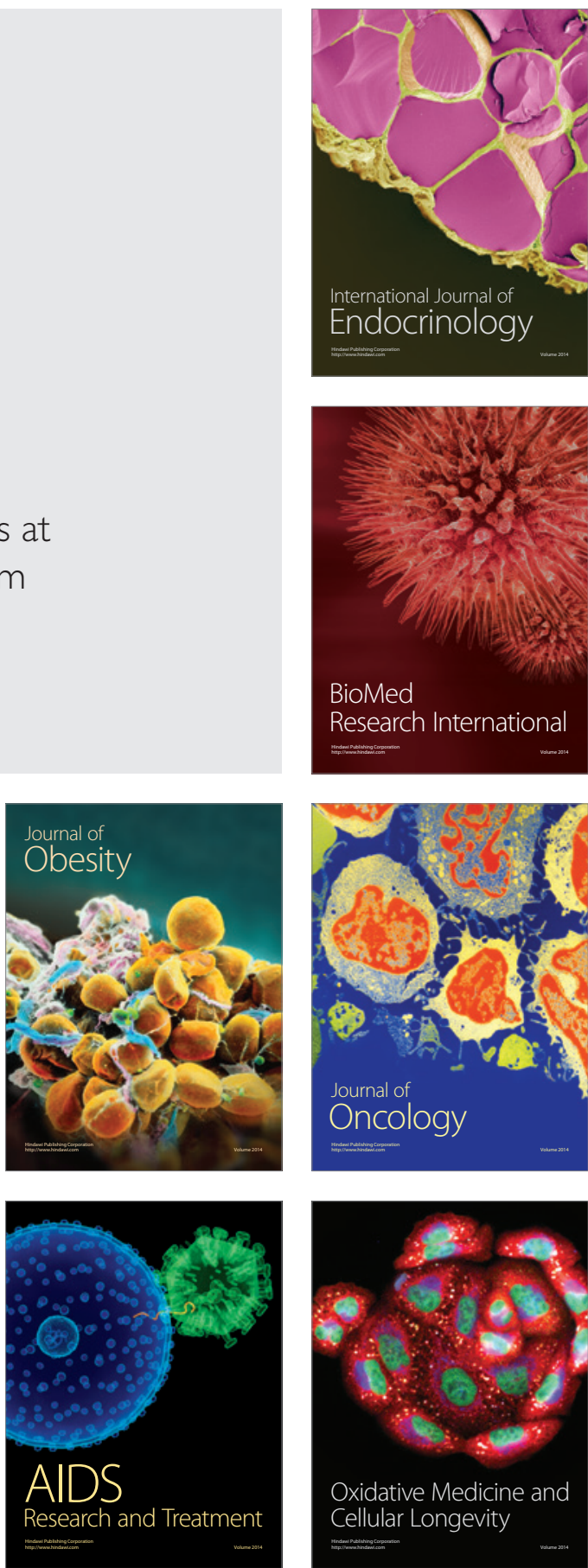